\title{
2. SYNOPSIS
}

\begin{tabular}{|l|l|}
\hline Name of Sponsor: I.R.I.S., 50 rue Carnot - 92284 Suresnes Cedex - France & (For National \\
\hline Test drug & Authority Use only) \\
Name of Finished Product: & \\
Not applicable & \\
Name of Active Ingredient: & \\
S49076 & Page: \\
\hline Individual Study Table Referring to Part of the Dossier & Volume: \\
\hline
\end{tabular}

Title of study: Phase I/II study of S49076, a multi-target inhibitor of c-MET, AXL, FGFR in combination with bevacizumab in patients with recurrent glioblastoma multiforme.

Protocol No.: CL1-49076-002

EudraCT No.: 2013-003079-37

The description of the study protocol given hereafter includes the modifications of the two substantial amendments to the protocol.

\section{International coordinator}

\section{Study centres (phase I part):}

Three centres located in 2 countries screened 18 patients and included 17 patients: 2 centres in France (14 patients included) and one centre in Switzerland (3 patients included).

Publication (reference): Not applicable

\section{Studied period:}

Initiation date: 06 October 2014 (date of first visit first patient)

Phase of development of the study:

Completion date: 14 November 2016 (date of last withdrawal visit

last patient)

\section{Objectives:}

This study was aimed to evaluate the safety and efficacy of S49076 in combination with bevacizumab in patients with recurrent glioblastoma multiforme (GBM). This was a phase I, dose-finding study of S49076 in combination with bevacizumab followed by a randomised efficacy phase II study.

\section{PHASE I: DOSE-FINDING PART}

The purpose of the dose-finding Phase I part was to determine the Maximum Tolerated Dose (MTD), the DoseLimiting Toxicities (DLTs) and to establish the recommended phase II dose (RPIID) of S49076 in combination with a fixed dose of bevacizumab in patients with recurrent GBM.

Primary objectives were to:

- Determine the safety profile and tolerability of S49076 given in combination with a fixed dose of bevacizumab in patients with recurrent GBM in terms of MTD and DLT (DLT, assessed during cycle 1).

- Establish the RPIID of S49076 given in combination with a fixed dose of bevacizumab.

Secondary objectives were to:

- Evaluate the pharmacokinetic (PK) profile of S49076 in combination with bevacizumab.

- Evaluate the pharmacodynamic (PD) effects of S49076 and bevacizumab using blood samples before and under treatment.

- Measure tumour response of S49076 in combination with bevacizumab by using RANO criteria (Response Assessment in Neuro-Oncology) to interpret repeated Gadolinium-Magnetic Resonance Imaging (Gd-MRI), neurological status and steroid dosing. 


\section{PHASE I: DOSE-FINDING PART (Cont'd)}

\section{Exploratory objectives were:}

- To evaluate the biomarkers potentially predictive of S49076 response using archived tumour.

- To assess antiangiogenic effects with Dynamic Susceptibility Contrast MRI (DSC-MRI).

- Optional part: to determine the S49076 levels and to evaluate the biomarkers of interest in the cerebrospinal fluid (CSF).

- Optional part: to perform a genomic biomarker analysis of inter patients variations in genes encoding for proteins involved in absorption/distribution/metabolism/excretion (ADME).

\section{PHASE II: EFFICACY PART}

The purpose of the efficacy phase II part was to evaluate the anti-tumour activity of S49076 given at the recommended dose defined in the phase I part with standard dose of bevacizumab in patients with recurrent GBM. The design planned to randomise patients with recurrent GBM in 2 arms to receive S49076 and bevacizumab (arm 1) versus bevacizumab alone (arm 2). In arm 2, patient with disease progression could cross-over to arm 1. The primary objective was to determine the PFS rate at 6 months according to RANO criteria.

It was a Sponsor decision not to continue the development of the S49076 in recurrent GBM indication beyond the phase I. This decision was related to the preliminary data which did not suggest an increase of activity by adding S49076 to bevacizumab in patients with recurrent GBM.

\section{Methodology (phase I part):}

The phase I part was an international, multicentre, open-label, dose-finding study, conducted among patients with recurrent GBM:

The dose-finding_followed a standard " $3+3$ " dose escalation designed to establish the MTD and the RPIID of the S49076 when combined with bevacizumab; the study was conducted in cohorts of 3 to 6 patients, with recurrent GBM, treated at increasing doses of S49076 in the range of 400 to $600 \mathrm{mg}$ in combination with a fixed dose $10 \mathrm{mg} / \mathrm{kg}$ of bevacizumab.

This study was performed in strict accordance with Good Clinical Practice including the archiving of essential documents.

\section{Number of patients (phase I part):}

Planned: up to 25 patients.

Included: 18 patients screened, 17 patients included, 16 patients treated (12 in dose escalation and 4 in expansion part).

\section{Diagnosis and main criteria for inclusion (phase I part):}

Male or female patients aged $\geq 18$ years with histologically confirmed diagnosis of GBM (WHO grade IV), with measurable tumour disease as defined by RANO (Response Assessment in Neuro-Oncology), unequivocal evidence of progression/recurrence after standard treatment with combined chemo-irradiation with temozolomide, and no more than one prior line of treatment. Estimated life expectancy $>12$ weeks. Karnofsky performance status $\geq 70 \%$. Adequate haematological, renal and hepatic functions.

\section{Test drug (phase I part):}

S49076 (hard gelatin capsule of $100 \mathrm{mg}$ ) was administered orally without food, on a continuous once daily $(q d)$ schedule of 28-day cycle in combination with bevacizumab infusion at a dosage of $10 \mathrm{mg} / \mathrm{kg}$ on D1, D15 of each cycle.

\section{Dose escalation of $\$ 49076$}

The dose escalation of S49076 followed a standard algorithm-based design " $3+3$ ". Each patient was observed for acute toxicity (DLT) for 28 days from the first day of treatment and a DLT assessment was performed after the completion of the first cycle.

A minimum of 3 patients who met eligibility criteria were enrolled at the starting dose level $1 \mathrm{of} 400 \mathrm{mg}$. A panel of 4 doses of $S 49076$ could be tested in combination with fixed dose of bevacizumab $(10 \mathrm{mg} / \mathrm{kg}) \mathrm{as}$ following: level 1 (starting dose) as $400 \mathrm{mg} q d$ /day, level 2 as $500 \mathrm{mg} q d /$ day and level 3 as $600 \mathrm{mg} q d /$ day. Dose de-escalation at level -1 as $300 \mathrm{mg}$ qd /day was planned if necessary. The dose of $600 \mathrm{qd} \mathrm{mg} / \mathrm{day}$ corresponded to the RD in the First In Human study in monotherapy; no upper dose was planned to be tested. The dose escalation only concerned S49076; no dose modifications of bevacizumab were permitted.

\section{Dose expansion of $\mathbf{S 4 9 0 7 6}$}

Once the MTD was reached, the RPIID level was expanded with additional patients, in order to reach 10 patients to complete safety and PK data. 


\section{Comparator (Reference product and/or placebo):}

None.

\section{Duration of treatment (phase I part):}

Treatment with S49076 and bevacizumab had to be initiated no later than 3 days after inclusion visit. Each patient had to receive the treatments until disease progression, unacceptable toxicity, or investigator's/patient's decision of withdrawal.

The maximum number of cycles was at the discretion of the investigator.

\section{Criteria for evaluation (phase I part):}

Efficacy measurements: antitumor activity based on the response assessment in RANO criteria.

\section{Safety measurements (primary objectives):}

Determination of the MTD, DLTs (assessed during cycle 1) and the adverse event (AE) profile of S49076 were the primary objectives as well as RPIID establishment.

Safety measurements were the following: DLT assessment, AEs and toxicity (grading according to the CTCAE $\mathrm{v} 4.0$ ), any change or addition of a new concomitant treatment, clinical neurological examination, physical examination including vital signs (temperature, weight, SDP, DBP, HR), Karnofsky Performance Status, laboratory tests (haematology, blood biochemistry, urinalysis and coagulation), 12-lead ECG (central reading), left ventricular ejection fraction (LVEF) at echocardiography or MUGA scan.

After the end of the participation in the study, during the post withdrawal follow-up, the date of progression and the patient's survival was recorded every 3 months up to 6 months.

\section{Pharmacokinetic (PK) measurements:}

- Blood samples concentrations of S49076 and its metabolites.

- CSF concentrations of S49076: planned but no CSF sample was collected (no medical indication).

\section{Pharmacodynamic (PD) measurements:}

- Genomic (circulating DNA) were planned on blood samples but no measurements were done.

- CSF concentrations of soluble growth factors and circulating: planned but no CSF sample was collected.

- Tumoral biomarkers: circulating blood proteins (HIF1a, MMP2, MMP9, PGF, VEGF) and biomarkers measured on tumour biopsy by IHC (MET, AXL, MER) and FISH (MET).

Pharmacogenomic (PG) measurements for translational research (optional samples):

Blood sample to interpret inter-patients PK variations in relation to polymorphisms of genes encoding for proteins involved in absorption/distribution/metabolism/excretion (ADME).

\section{Statistical methods (phase I part):}

Analysis Sets:

- Included Set (IS): all included patients.

- Safety Set (SS): all patients who received at least one dose of S49076 or bevacizumab.

- Full Analysis Set (FAS): based on the intention-to-treat principle, this set corresponded to included patients having taken at least one dose of S49076 or bevacizumab, and without any relevant deviation at baseline.

- Response Evaluable Set (RES): all patients in the FAS having at least one baseline and one post-baseline tumour evaluation according to the RANO criteria.

- Dose Limiting Toxicity Evaluable Set (DLTES): all patients from SS who were evaluable for DLT according to the DLT assessment at end of cycle 1. A patient wasn't considered evaluable if:

- $\mathrm{He} / \mathrm{she}$ discontinued during first cycle for a reason other than DLT or

- $\mathrm{He} /$ she did not undergo a DLT assessment at the start of cycle 2 or

- $\mathrm{He} / \mathrm{she}$ did not receive at least 85\% (24 doses 28) of S49076 doses, unless treatment was stopped for a DLT.

- $\mathrm{He} / \mathrm{she}$ did not receive the two infusions of bevacizumab, unless treatment was stopped for a DLT.

Study outcome: descriptive statistic by dose level and overall for disposition, extent of exposure and treatment compliance. Descriptive statistic in the $\mathrm{RD}$ and overall for baseline characteristics, corticosteroids and concomitant treatments. 
Efficacy analysis was carried out on the FAS. The best overall response (BOR) was provided for each dose level, whereas the Objective Response Rate (ORR), Clinical Benefit Rate (CBR), duration of clinical benefit, duration of response, overall survival (OS), OS rate at 6 months, time to first response and the Progression Free Survival (PFS) were provided in tables and/or graphs for the RD and overall. All dose levels were presented in listings. The overall response was evaluated according to the investigator assessments. The survival functions of the time dependent parameters (duration of clinical benefit, duration of response and PFS) were estimated via Kaplan-Meier curves.

Safety analysis (primary objectives): description of DLTs on the DLTES for dose escalation and expansion parts. Description of emergent adverse events, clinical laboratory evaluation, vital signs, neurological examination (no planned analysis), LVEF and ECG parameters on the SS; by dose level and overall.

Pharmacokinetic analysis: see separate PK report.

\section{Pharmacodynamic analysis:}

Circulating blood proteins: relative change from baseline and C2D1 according to response and dose levels.

Biomarkers IHC (Hscore membrane and cytoplasm) and FISH (amplification status) according to response.

Pharmacogenomic analysis: see separated bio-analytical report.

\section{SUMMARY - CONCLUSIONS}

The results presented below only concerned the dose-finding Phase I part of the study.

\section{DISPOSITION OF PATIENTS AND ANALYSIS SETS}

A total of 18 patients were screened, 17 were included and 16 treated. Two patients were still on-going in the follow-up period at the time of the present main analysis. Data not available will be presented in an addendum to this clinical study report. Number of patients in each analysis sets are presented in table below.

Table 1 - Analysis Sets

\begin{tabular}{lcc}
\hline & & All \\
\hline Included & $\mathrm{n}$ & $\mathbf{1 7}$ \\
Withdrawn due to & $\mathrm{n}$ & $\mathbf{1 7}$ \\
- progressive disease & $\mathrm{n}(\%)$ & $13(76.5)$ \\
- adverse event & $\mathrm{n}(\%)$ & $3(17.6)$ \\
- non-medical reason & $\mathrm{n}(\%)$ & $1(5.9)$ \\
Safety set & $\mathrm{n}(\%)$ & $\mathbf{1 6}(\mathbf{9 4 . 1 )}$ \\
DLT Evaluable Set & $\mathrm{n}(\%)$ & $\mathbf{1 5}(\mathbf{8 8 . 2 )}$ \\
FAS & $\mathrm{n}(\%)$ & $\mathbf{1 6}(\mathbf{9 4 . 1 )}$ \\
\hline$\%$ was of included patients; RES was identical as FAS
\end{tabular}

\section{BASELINE CHARACTERISTICS}

Patients enrolled in the study $(\mathrm{N}=17)$ presented a recurrent GBM grade IV and were mainly male $(70.6 \%)$ with median age of 57 years. At initial diagnosis 16 patients presented de novo grade IV astrocytoma (14 glioblastoma, 1 glioblastoma with oligodendroglioma component, 1 gliosarcoma) and one patient presented a mixed low grade III with secondary transformation in GBM.

At inclusion in the study, GBM was initially diagnosed since $1.6 \pm 1.5$ years in average (median $=1.0$ years) and its recurrence was diagnosed since $1.1 \pm 0.9$ months in average (median $=0.9$ month). Surgical resection had been performed for 14 patients (total removal: $n=9$; partial removal: $n=5$ ) at initial diagnosis and for 3 patients at recurrence (total removal: $\mathrm{n}=1$; partial removal: $\mathrm{n}=2$ ).

All patients had received one prior line of treatment for GBM: combination of radiotherapy and concomitant or adjuvant temozolomide i.e. protocol Stupp $(\mathrm{n}=14)$, combination of radiotherapy and concomitant temozolomide $(\mathrm{n}=2)$ or protocol Stupp + Novocure $(\mathrm{n}=1)$.

Karnofsky status was $\geq 70$ for all the patients and $\geq 90$ for half of the patients. Baseline characteristics of patients at RD $600 \mathrm{mg} q d$ were comparable to those of overall patients.

\section{EXTENT OF EXPOSURE}

In the Safety Set, the median treatment duration was 16.1 weeks (from 9.9 to 22.5 weeks). The median number of cycles was 4.0 cycles (from 2 to 11 cycles). 


\section{EFFICACY RESULTS}

Among the 16 patients of the FAS, the BORs were confirmed partial response in 6 patients $(37.5 \%)$, stable disease in 8 patients $(50 \%)$, and progressive disease in 2 patients $(12.5 \%)$. The median time to first response was 8.1 weeks with a median duration of response of 18.2 weeks. The objective response rate was $37.5 \%$. The clinical benefit rate was $44 \%$ with a median duration of 27.7 weeks. The median PFS was 16.3 weeks. The median overall survival (OS) was 64.4 weeks and the survival rate at 6 months was $87.5 \%$. Results were similar in patients at RD except for OS which was lower (median $=39.1$ weeks) than in all patients.

These results were in the range of those reported in literature with bevacizumab alone and did not suggest an increase of activity by adding S49076 to bevacizumab.

Regarding biomarkers, specific serum proteins were significantly increased during treatment between baseline and cycle 2 pre-dose with an estimate of $143 \%$ for PGF, $82 \%$ for HIF1a and $19 \%$ for MMP2 while it significantly decreased with an estimate of $-77 \%$ for VEGFA. No dose effect neither effect according to response was observed. A trend to higher levels at baseline in responders was observed for MMP9 and VEGFA but less marked for the latter. Only 1 high and 1 intermediate amplification status was observed for MET biomarker in non-responder patients. No obvious difference according to response was observed for any biomarkers explored.

Based on these efficacy and pharmacodynamic results, the phase II part of the study was not initiated.

\section{SAFETY RESULTS}

\section{Dose-escalation, MTD, RPIID and PK finding}

DLTs were assessed during cycle 1 according to a " $3+3$ " design. The dosages tested were $400 \mathrm{mg} q d, 500 \mathrm{mg}$ $q d$ and $600 \mathrm{mg} q d$. One DLT, asymptomatic LVEF decrease grade 3, was observed at $600 \mathrm{mg} /$ day among 6 patients. At the end of dose escalation, no MTD was reached and the RPIID was defined as $600 \mathrm{mg} q d$. Four additional patients were included during dose expansion, so up to 10 patients could confirm the RPIID, and complete the safety and PK data. No new DLT was observed in the patients of the dose expansion part and confirmed the good tolerability of this dose level.

The global safety profile observed in the 16 patients treated with the combination S49076/bevacizumab was comparable to the one observed in the first-in-human study.

PK data showed: rapid absorption ( $\mathrm{T}_{\max } \approx 3.0$ to $4.0 \mathrm{~h}$ ) and moderate to high interindividual variability for $\mathrm{C}_{\max }$ and $\mathrm{AUC}_{24}$ on Day 1 and Day 28. In this study, exposures and $\mathrm{C}_{\max }$ seemed to increase proportionally to the dose on Day 1. The results should be interpreted with caution due to the small number of patients and the moderate to high variability of $\mathrm{C}_{\max }$ and $\mathrm{AUC}_{24}$ on Day 1 and Day 28.

Based on safety and PK data, the RPIID of S49076 given in combination with a fixed dose of bevacizumab was confirmed as $600 \mathrm{mg} q d$. 


\section{SAFETY RESULTS (Cont'd)}

\section{Emergent adverse events}

Main results for AEs in the Safety Set are described in Table 2.

Table 2: Overall summary for adverse events in the Safety Set

\begin{tabular}{lcc}
\hline & & $\begin{array}{c}\text { All } \\
(\mathbf{N}=\mathbf{1 6})\end{array}$ \\
\hline $\begin{array}{l}\text { Patients having reported } \\
\text { at least one EAE }\end{array}$ & $\mathrm{n}(\%)$ & $16(100)$ \\
at least one S49076 only-related EAE & $\mathrm{n}(\%)$ & $16(100)$ \\
at least one bevacizumab only-related EAE & $\mathrm{n}(\%)$ & $10(63)$ \\
at least one S49076 and bevacizumab related EAE & $\mathrm{n}(\%)$ & $8(50)$ \\
at least one severe EAE & $\mathrm{n}(\%)$ & $10(63)$ \\
Patients having experienced & & \\
at least one serious AE & $\mathrm{n}(\%)$ & $8(50)$ \\
at least one serious EAE & $\mathrm{n}(\%)$ & $7(44)$ \\
at least one S49076 only-related serious EAE & $\mathrm{n}(\%)$ & $1(6)$ \\
at least one bevacizumab only-related serious EAE & $\mathrm{n}(\%)$ & $1(6)$ \\
at least one S49076 and bevacizumab related serious EAE & $\mathrm{n}(\%)$ & $1(6)$ \\
Patients with treatment withdrawal & & \\
due to an EAE & $\mathrm{n}(\%)$ & $2(13)$ \\
due to a serious EAE & $\mathrm{n}(\%)$ & $2(13)$ \\
due to a S49076 only-related serious EAE & $\mathrm{n}(\%)$ & - \\
due to a bevacizumab only-related serious EAE & $\mathrm{n}(\%)$ & - \\
due to a S49076 and bevacizumab related serious EAE & $\mathrm{n}(\%)$ & $1(6)$ \\
Patients who died & & \\
during the treatment period & $\mathrm{n}(\%)$ & - \\
during the follow-up period & $\mathrm{n}(\%)$ & $7(43.8)$ \\
\hline
\end{tabular}

Overall, the most frequently reported EAEs were peripheral oedema (56\%), diarrhoea (50\%), epistaxis (44\%), asthenia (38\%), hypoalbuminaemia (31\%), dry skin (31\%), ALT increased $(31 \%)$ and AST increased $(31 \%)$. Most EAEs (80\%) recovered. Overall, most of EAEs $(86 \%)$ were grade 1 or 2 and 13\% of EAEs grade 3 or 4 (in 10 patients).

Overall, 102/210 EAEs were considered by the investigators to be related to $\mathbf{S 4 9 0 7 6}$ or/and bevacizumab:

- 66 EAEs in 16 patients (100\%) were considered as related to S49076 only. The most frequent ( $>2$ patients) were peripheral oedema $(n=8)$, hypoalbuminaemia $(n=5)$, ALT increased $(n=5)$, AST increased $(n=5)$, dry skin $(n=4)$, diarrhoea $(n=4)$, yellow skin $(n=3)$ and platelet count decreased $(n=3)$. Oedema peripheral and hypoalbuminaemia were in accordance with a possible MET-inhibitor class effect.

- 21 EAEs in 10 patients $(63 \%)$ were considered as related to bevacizumab only. The most frequent were epistaxis $(n=7)$ and hypertension $(n=3)$, in accordance with bevacizumab known safety profile.

- 15 EAEs in 8 patients (50\%) were considered as related to S49076 and bevacizumab. None occurred in more than 2 patients.

Most of the treatment-related EAEs were grade 1 or 2: $97 \%$ of S49076 related EAEs, $81 \%$ of bevacizumab related EAEs and $73 \%$ of both S49076 and bevacizumab related EAEs.

Four EAEs led to treatment withdrawals in 2 patients and were all serious EAEs (SEAEs).

Overall, 8 patients (50\%) experienced 20 serious adverse events during the study including 18 serious SEAEs in 7 patients (44\%). One SEAE (left ventricular ejection fraction decreased grade $3=$ DLT) was considered as related to S49076 only, one SEAE (deep vein thrombosis grade 2) as related to bevacizumab only and 3 SEAEs (abdominal abscess, diverticulitis and large intestine perforation, all grade 2) in one patient were considered related to S49076 and bevacizumab.

Among the 4 SEAEs leading to treatment withdrawal, 3 were those mentioned previously as related to S49076 and bevacizumab and 1 was subclavian vein thrombosis grade 2 (not treatment-related).

No death occurred during the treatment period. After the withdrawal visit i.e. during the follow-up period, seven patients died for progression of the studied tumour. 


\section{SAFETY RESULTS (Cont'd)}

\section{Blood laboratory evaluation}

For the biochemistry parameters, the most frequently observed emergent abnormal values were low total protein (11 patients, $69 \%$ ) which was consistent with similar percentage of patients $(69 \%)$ who had emergent abnormal low albumin values (all grade 1 or 2). Among gradable biochemical parameters, one patient experienced emergent grade 3 high GGT reported as EAE 'GGT increased', not serious and not treatment related.

For the haematological parameters, 4 severe emergent abnormal values were reported in 3 patients: 1 patient for low lymphocyte and low white blood cell (both grade 3) reported as EAE 'lymphocyte count decreased', not serious and not treatment related; 1 patient for grade 3 low lymphocyte reported as EAE 'lymphocyte count decreased', not serious and related to S49076; 1 patient for grade 4 low lymphocyte reported as EAE 'lymphopenia' not serious and not treatment related.

\section{Other safety evaluation}

No relevant change in blood pressures and heart rate were observed. A median weight increase of $5 \mathrm{~kg}$ was observed, the increases being generally concomitant to adverse events peripheral oedema (present in $56 \%$ of the patients).

No ECG abnormality was detected on treatment. All patients had QTcF value $\leq 450 \mathrm{~ms}$. Maximum QTcF change from baseline was $\leq 30 \mathrm{~ms}$ for all patients, except 2 patients within ]30-60] ms.

A LVEF decrease greater or equal to $10 \%$ was observed in 3 patients on treatment. Two LVEF decrease in 2 patients were reported as EAE. One was non-serious (grade 2), the other serious (grade 3, DLT). Both were asymptomatic, considered as S49076 related and recovered. The third patient showed an $11 \%$ LVEF decrease (from 73 to $62 \%$ ) without EAE reported.

Most of patients (81\%) had unchanged Karnofsky Performance Status class on treatment.

\section{CONCLUSION}

This phase I/II study aimed to evaluate the safety and efficacy of S49076 in combination with bevacizumab in patients with recurrent glioblastoma multiforme (GBM).

The dose-finding phase I part was to determine safety profile and recommended dose (RD) of S49076 in combination with bevacizumab in patients with recurrent GBM.

In this phase I, a total of 16 patients were treated. The $S 49076$ dosages tested were $400 \mathrm{mg} q d, 500 \mathrm{mg} q d$ and $600 \mathrm{mg} q d$. One patient experienced a DLT at $600 \mathrm{mg} q d$, an asymptomatic left ventricular ejection fraction decrease grade 3. Based on safety and PK data, the RD of S49076 in combination with infusions of bevacizumab at $10 \mathrm{mg} / \mathrm{kg}$ on days 1 and 15 during 28-day cycles was determined as $600 \mathrm{mg} q d$. No maximum tolerated dose was reached.

The most frequent emergent adverse event considered as related to $\mathbf{S 4 9 0 7 6}$ only was peripheral oedema, as similarly observed in first-in-human study, followed by hypoalbuminemia, ASAT and ALAT increased. The tolerability profile of the combination was similar to that observed in the first-in-human study in monotherapy.

The best overall response was confirmed partial response in $37.5 \%$ of patients and stable disease in $\mathbf{5 0} \%$. The objective response rate was $37.5 \%$. The clinical benefit rate was $44 \%$ with a median duration of 27.7 weeks. The median progression free survival was 16.3 weeks. The median overall survival was 64.4 weeks and the survival rate at 6 months was $87.5 \%$. These results were in the range of those reported in the literature with bevacizumab alone and did not suggest an increase of activity by adding S49076 to bevacizumab. No obvious difference according to response was observed for any biomarkers explored.

Based on these efficacy and pharmacodynamic results, the phase II part of the study was not initiated.

Date of the report: 18 July 2017

Version of the report: Final version 